\title{
Development of Computer Graphics
}

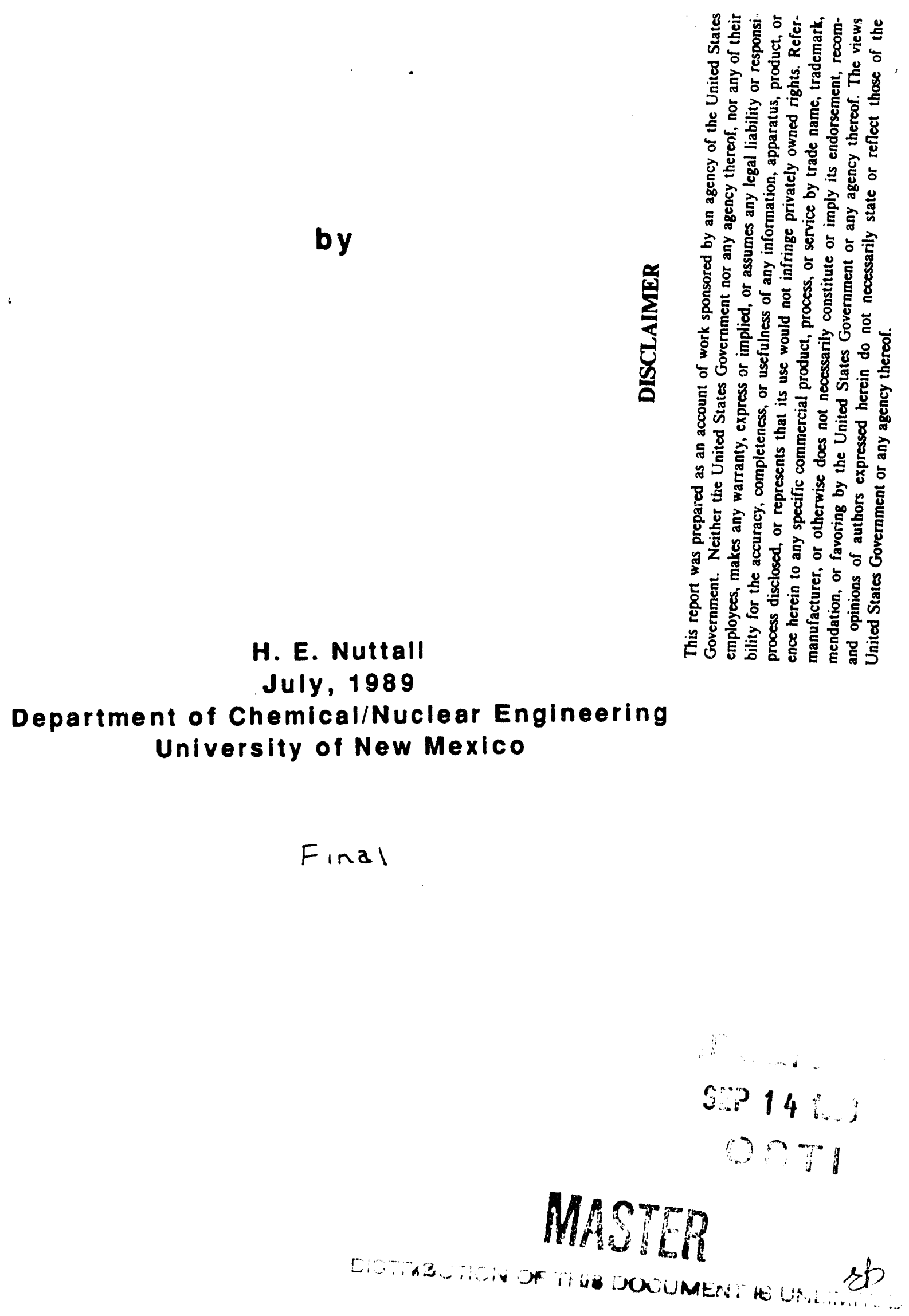


Table of Contents

Abstract

I. Introduction

A. Purpose

B. Accomplishments

1. Screening of the Three Packages

2. Acquisition, Implementation, and Testing of NCSA Image

3. Mac II Setup and Networking

II. Installation and Operation of NCSA Image.

A. FTP acquisition of NCSA routines Macll and Sun 4

111. Description of NCSA Image for the Macll and Examples
A. Ted's test image sequence
B. Production of $35 \mathrm{~mm}$ slides
C. Production of video recorded animated images
D. HDF Installation and Use
E. Plume Animation

V. Appendices
A. NCSA Image Manual
B. DataScope Manual
C. NCSA Layout
C. HDF Manual
D. HDF Manual-Specific
E. Palette Manual
F. Telnet Manual
G. HDF (Raster.f) 
Abstract

The purpose of this project was to screen and evaluate three graphics packages as to their suitability for displaying concentration contour graphs. The information to be displayed is from computer code simulations describing air-born contaminant transport. The three evaluation programs were MONGO (John Tonry, MIT, Cambridge, MA, 02139), Mathematica (Wolfram Research Inc.), and NCSA Image (National Center for Supercomputing Applications at the University of Illinois at Urbana-Champaign). After a preliminary investigation of each package, NCSA Image appeared to be significantly superior for generating the desired concentration contour graphs. Hence subsequent work and this report describes the implementation and testing of NCSA Image on both an Apple Macll and Sun 4 computers. NCSA Image includes several utilities (Layout, DataScope, HDF, and PalEdit) which were used in this study and installed on Dr. Ted Yamada's Mac II computer. Dr. Yamada provide two sets of air pollution plume data which were displayed using NCSAImage. Both sets were animated into a sequential expanding plume series. 


\section{Introduction.}

\section{A. Purpose}

The purpose of this task was to screen and evaluate three graphics packages capable of displaying concentrations as a function of $x-y$ spatial locations. The information to be displayed is from computer code calculations describing the transport of air-born contaminants. The three graphics packs evaluated are MONGO (John Tonry, MIT, Cambridge, MA, 02139), Mathematica (Wolfram Research Inc.), and NCSA Image (National Center for Supercomputing Applications at the University of Illinois at UrbanaChampaign). After a preliminary investigation of each package, NCSA Image appeared to be the stronger utility for the desired displays. Hence this report describes the implementation and testing of NCSA Image on both an Apple Macll and Sun 4 computers.

\section{Description of MONGO}

MONGO is designed to produce high quality graphics output with a minimum of effort. It runs both interactively, interpretively, and in batch as calls to FORTRAN subroutines. MONGO consists of a number of subroutines designed to make $X-Y$ and shaded contour graphs. It was written and is currently distributed by John L. Tonry (MI, 1987). LANL has a sit license. It runs under VMS and BSD 4.2 UNIX. Currently it is running on the ESS-5 Sun-4 server RIGEL. MONGO is not as advanced as either Mathematica or NCSA Image. MONGO is not capable of 3D graphics, color, or animation.

\section{Description of Mathematica}

This Mac II program is primarily a symbolic manipulator with advanced graphics output. It was developed and is sold by Stephen Wolfram, (Wolfram Research, Inc.) and support 3D, color, contours, and animation. Since it is primarily a symbolic manipulator rather than a graphics tool, I decided to focus my development and testing of NCSA Image. Although the two programs a similar graphics capabilities.

Description of NCSA Image

This software is free to the general public. It was developed under an Nation Science Foundation grant and the project is continuing to be funded. 
The advanced graphics package called NCSA Image and the associated utilities (DataScope, NCSA Layout, HDF and Palette) were acquired from the National Center for Supercomputing Applications at the University of Illinois at Urbana-Champaign and implemented on both an Apple Mac II and a Sun 4 computers. For this study the latest versions of the software and manuals were electronically transferred from the University of Illinois at Urbana-Champaign using FTP. This transfer method is described in section II, Installation and Operation of NCSA Image. All of the NCSA graphics packages have user's manuals. The latest versions of these manuals may be found in the Appendices. Implementation and testing of the NCSA graphics was performed using a Mac II. The Sun 4 version of NCSA was loaded onto the system and tested briefly; however, NCSA is focusing its development on the the Mac II hardware at this time. They are developing an X-window version of NCSA Image to be released perhaps in 1990. Many of the ancillary utilities are not available for the Sun 4 computer system.

NCSA Image allows one to use contour plots, 3D plots, Shaded data plots, and dither plots to visualize sets of data points. In addition, $X Y$ graphs, a histogram, and animations may be generated from your dataset to allow advanced analysis of the image data. A copy of the user's manual is contained in Appendix $A$.

Additional utility codes which support and extend the usefulness of NCSA Image are DataScope, NCSA Layout, and HDF. Briefly DataScope is a complete Mac II only utility that permits the user to view either text or raster data in a spread sheet type format and at the same time in another window see the generated contour image of the data. The Manual for Datascope is presented in Appendix B. NCSA Layout is a Mac II only utility for annotating and adding axes to contour images (See Appendix $C$ for the User's Manual). Inaddition several images can be combined into a single frame for a time-sequence view of the data images.

The Hierarchical Data Format (HDF) is the advanced data/image format and file generating utilities developed by NCSA as a standard for all of their utilities (See Appendix D for the HDF User's Manual). NCSA found it necessary to develop their own data file format. NCSA Image can read either raster data or HDF data files but CAN NOT read ASCII text files directly. Only the DataScope utility can read the ASCII text files directly and save them in HDF format. However a new line command utility called tp_to_hdf is now available for the Sun 4. To implement this concept of HDF files, they wrote a series of Fortran or C-code callable subroutines designed to convert raw data into raster image formats directly within 
one's application code. A library of compiled subroutines must be created at the user's facility. A C-compiler is required. The necessary library, libdf.a, was created on both rigel and algol.

Also included in the appendices are utilities to create and edit palettes (Appendix E) and the terminal emulation routine Ielnet2.2 (Appendix F).

II. Installation and Operation of NCSA Image

A. FTP acquisition of NCSA routines Macll and Sun 4

The example shown below illustrates how NCSA software is acquired electronically (for either the Macll or Suns).

Example for using FTP

To logon the NCSA anonymous computer type the following commands:

fte 128.174 .20 .50

usr: anonymous

pass:login name on route machine

The following script is a sample interactive session on NCSA Anonymous:

Is

<PORT command successful.

<Opening ASCII mode data connection for $/ \mathrm{bin} / \mathrm{ls}$ ( 0 bytes).

<Transfer complete.

PalEdit.doc.sit.hqx

PalEdit.proj.sit.hqx

PalEdit.sit.hqx

README

64 bytes in 1 seconds -512 bps

" get README

<PORT command successful.

$<$ Opening ASCII mode data connection for README (368 bytes).

$<$ Transfer complete.

359 bytes in 1 seconds -2872 bps

"get PalEdit.sit.hax

<PORT command sucressful.

<Opening ASCll mode data connection for PalEdit.sit.hqx (175059 bytes).

<Transfer complete.

172363 bytes in 34 seconds -40552 bps 
" get PalEdit.dec sit hax

<PORT command suricessful.

<Opening ASCll mode data connection for PalEdit.doc.sit.hqx (65927 bytes).

<Transfer complete.

64910 bytes in 11 seconds -47200 bps

" get PalEdit.proj.sit.hax

<PORT command successful.

<Opening ASCII mode datá sonnection for PalEdit.proj.sit.hqx (180976 bytes).

$<$ Transfer completc.

178189 bytes in 38 seconds- -37512 bp's

Once an application program is acquired in this method is must be processed with both Binhex and Unstuffit software utilities. These utilities are installed on Dr. Yamada's Macll. Once the NCSA applications have been processed, they may be operated in accordance with the instructions provided in the Appendices.

III. Description of NCSA Image for the Macll and Examples

\section{A. Ted's test image sequence}

Initially Dr. Yamada provided a series of six images $(81 \times 81)$ to test and development the NCSAlmage software. A set of NSCA readable raster image files were created using my raster.f code. These images were later used in NCSALayout to product $35 \mathrm{~mm}$ slide using in addition the Montage software and slide equipment. The slides were given to Dr. Yamada.

Below in Figures 1-5 are a series of sin-cos function examples which illustrate the various display modes available in NCSAlmage.

Examples of the Sin-Cos function

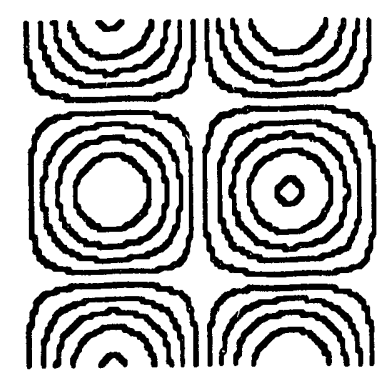

Figure 1. Contours 


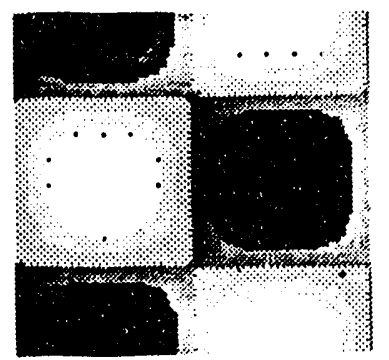

Figure 2. Shaded

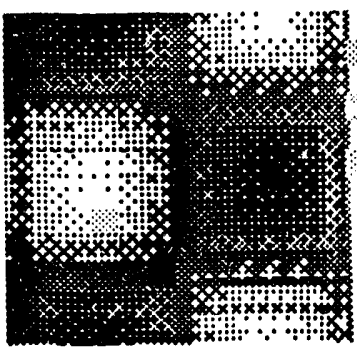

Figure 3. Dithered

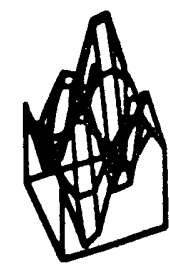

Figure 4. 3D Surface

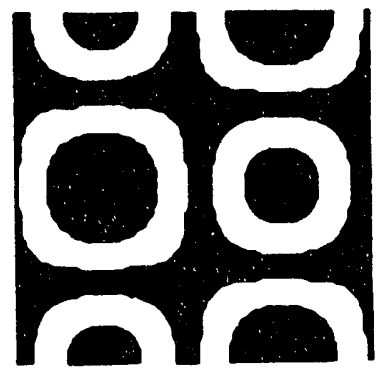

Figure 5. Copy of Color Contours

B. Production of $35 \mathrm{~mm}$ slides

Images for NCSALayout were saved in PICT2 formation and converted to $35 \mathrm{~mm}$ slides using the Montage software and hardware. The Montage unit with software costs about $\$ 5,000$ and makes excellent quality color 
slides and interfaces well with image from the NCSA product. For addition information on this product contact Computer One, 3401 Candelaria, NE, Albuquerque, NM 87107 at (505) 884-6610 or the Montage company:

Presentation Technologies

743 North Pastoria Avenue

Sunnyvale, CA 94086

C. Production of video recorded animated images

During June, 1989 and with the aid of Computer One, an Apple dealer in Albuquerque, a VHS video was produced. I used a Mass Color Space II card and software by Mass Mcirosystem (550 Del Rey Avenue, Sunnyvale, California 94086, phone 1-800-522-7979). The video showed and animation of the turbulent jet stream. This the standard example provided with NCSAlmage. The video quality was only fair. The 60 cycle scrolling was absent but the picture showed some distracting flickering. The VHS tape was reviewed by Dr. Ted Yamada. A single frame of the turbulent jet is shown below.

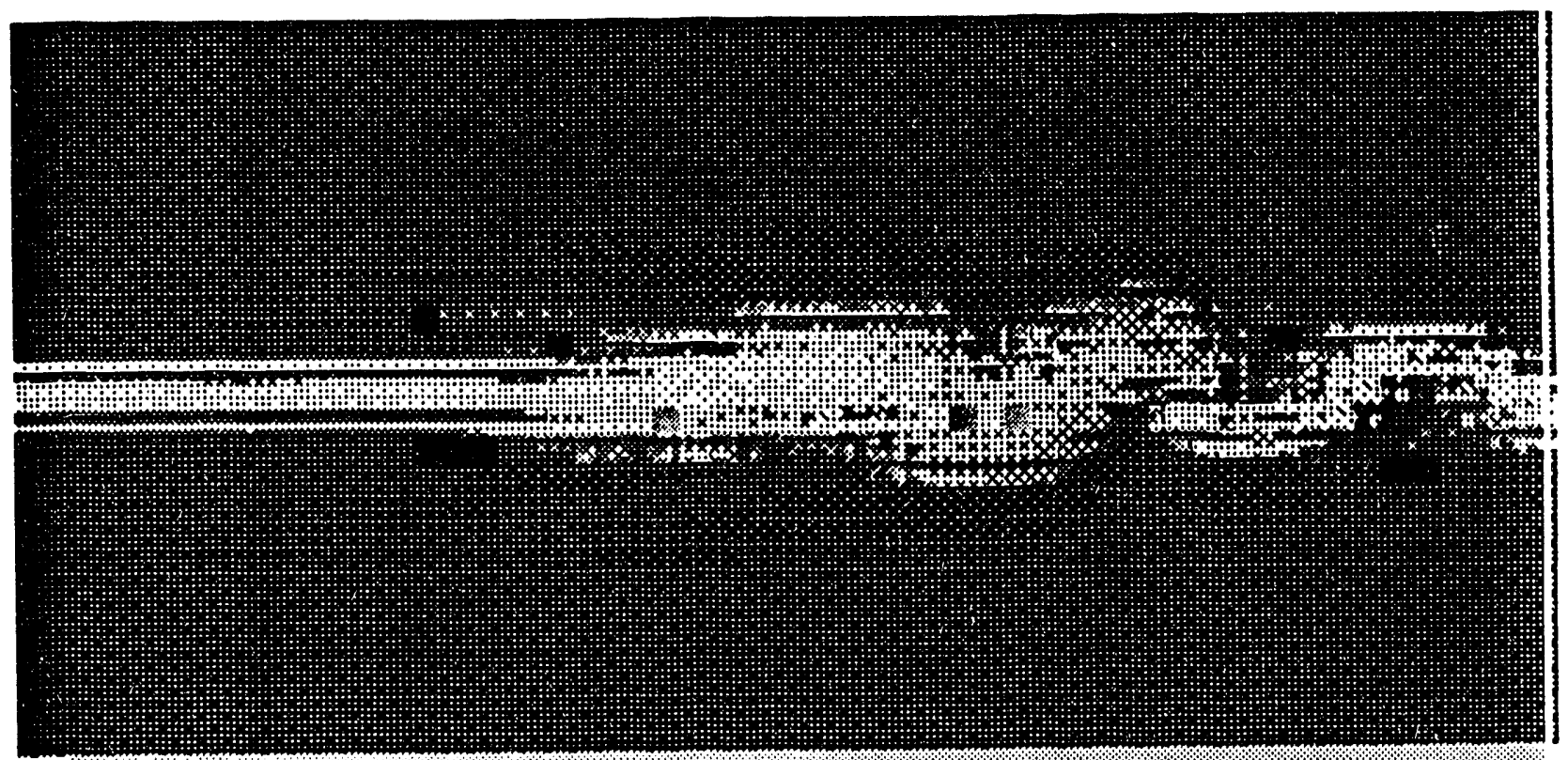

Figure 6. Turbulent Jet

The resulting video did show some disturbing flickering which is largely a result of viewing the Mac window opposed to the just the window contents. A new software product called Macro Mind Director will eliminate this problem by allowing a expanded display of the window 
so one only view the window contents on the screen. Recently I talked to the Mass Microsystems' technical support and they were aware of the problem and suggested using Macro Mind Director software to minimize the flickering.

D. HDF- Hierarchical Data Format

NCSA HDF is the Hierarchical Data Format, a standard file format developed by NCSA. The purpose of these subroutines are to facilitate the creation of graphic image files which in turn my be read directly with the NCSAlmage software. A compiled version of these files were placed on Dr. Yamada's Sun 4 ("algol"). Documentation on the use of HDF files is give in the Appendices. The files were transferred electronically form NCSA anonymous server (128.174.20.50). Procedures for receiving codes was described previously or may be found in the Appendices.

Included in this version are: the basic low-level routines to perform $1 / 0$ to HDF files, routines to implement the 8-bit Raster Image Set, which will read and write 8 -bit raster images and routines to implement the Scientific Data Set.

The SUN version files are:

Files: (description of files in each source directory) df.h - header file included in all HDF source files and user programs dfi.h - internal header file, included automatically by $d f . h$ df.c - low-level routines which perform I/O to HDF files etc. dfrig.h - header file for the Raster Image Group set dfr8.c - routines to implement 8 -bit raster image groups dfgroup.c - routines to read and write groups (of tag/refs) dfcomp.c - routines for data compression dfimcomp.c - routines to implement the IMCOMP compression scheme dfF.h - header file for user FORTRAN routines dfF.c - routines which translate Fortran calls to $C$ routines in df.c dfFf.f - Fortran stubs to translate Fortran calls to $C$ stubs in dif.C dfr8F.c - routines which translate Fortran $R 8$ calls to $C$ routines in dfr8.c

dfr8Ff.f - Fortran stubs to translate Fortran R8 calls to $C$ stubs in dfrif.c

dfsd.c - routines to implement the Scientific Data set 
dfsd.h - header file for the Scientitic Data set

dfsdF.c - routines which translate Fortran SD calls to $C$ routines in dfsd.c

disdFf.f - Fortran stubs to translate Fortran SD calls to $C$ stubs in dfsdF.c

dfkit.c - HDF internal utility routines

hdfls.c - source for "hdfls" which lists contents of HDF files

hdfrseq.c - source for "hdfseq" which displays images in HDF files and

"hdfrseq" which produces ICR output for display on remote screen

r8tohdf.c - source for "r8tohdf" which converts raw image, palette files

to HDF

Makefile - makefile for the HDF system

HDF files were generated from a raw floating point file and then using FTP (binary mode) they were downloaded to the Macll where they were viewed using NCSAlmage. A Fortran code, raster.f, was written to perform the file conversion and test the HDF subroutines. The code and procedure performed the task of generating HDF files on the Sun 4 and then rapidly transferring them to the Macll where they could be displayed. The raster.f code is listed in the Appendix.

\section{E. Plume Animation}

In this section I described the development of a second animation using data provided by Mike Williams taken from code simulations generated on the Sun 4, Algol, at Los Alamos National Laboratory. The objective was to develop a plume expansion animation with topological contours overlayed upon the plume. To accomplished this task four NCSA routines were used ( $f p$ to_hdf, NCSA Telnet, PalEdit, NCSAImage, and NCSALayout). Six sequential ascll data files were generated by Mike Williams and transferred to my account under the subdirectory movie. There was an additional file containing the topographical data thus seven files in all. The 2D data matrix was $81 \times 81$. The data was written in the standard NCSA text format as described below:

nrows ncols

max_value min_value

row 1 row2 row3 row 4 ......

col1 col2 col3 col4 ......

data1 data2 data3 .... 
(row1, row2, etc., and col1, col2, etc., are scales for the vertical and horizontal axes. There are nrows vertical axis values and ncols horizontal axis values. $i$

Data1, data2, etc., are the floating point data and are assumed to be stored in column major order.)

Next the seven data files (six plume and one contour) were converted to HDF format by using the NCSA line command utility fp_to_hdf. Please refer to the HDF Appendix in this report for detailed information on the use of fp_to_hdf. An insert on $f p_{-}$to_hdf is presented in the front of this appendix. Now the seven files are in binary format on Algol and next were downloaded to the ESS-5 Macll using ftp under Telnet (please refer to the Telnet Appendix for detailed instructions on the use of ftp-file transfer protecol). Once the seven files are downloaded to the Macll, the animation sequence of six frames was generated using both NCSAlmage and NCSALayout in unison, i.e., both programs needed to be running on the Macll and one must switch back and forth. The composite plume/contour animation images were created in NCSALayout. The first step in this custom animation was to create the desired plume images which required a special palette. The utility PalEdit was used to modify the NCSA default palette by changing the low end purple to white. Next with both NCSAImage and NCSALayout running, frame by frame the plume images were moved from NCSAImage to NCSALayout. For each frame, the identical ten level contour image was superimposed over the plume. Each frame was save in sequence in a special folder. After a frame was generated and saved the plume image was deleted and the next plume image copied in or loaded in (Load Image under File). The key issue is that the NCSALayout window must remain open and at exactly the same size during the development of all six frames. There are two file formats that that are suitable for storing the frames. Either the HDF format which is created by SELECTING WINDOW under the Edit and the SAVE SELECTION under File. The file icons are different for each format. The animations are actually run using NCSAlmage. The following figure is a summary of the animation generation procedures. 


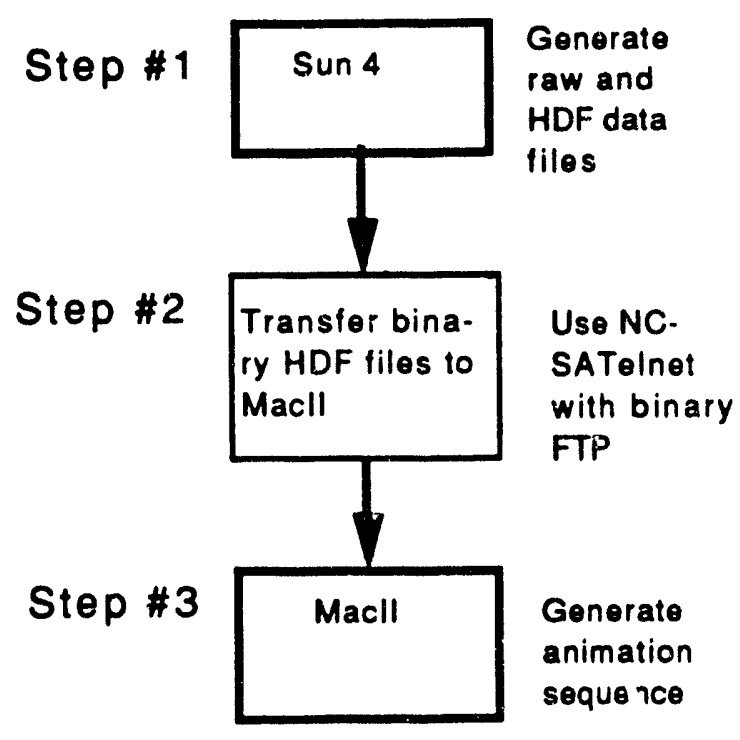

Run animation using

NCSAlmage

Figure 7. Animation Procedures

The animation can also be copied to VHS video using the ColorSpace II software and hardware system plus a recording device such as a camcorder or VHS video recorder. 
Shown in Fig. 8 is a sample frame from the final plume animation.

$\therefore !$

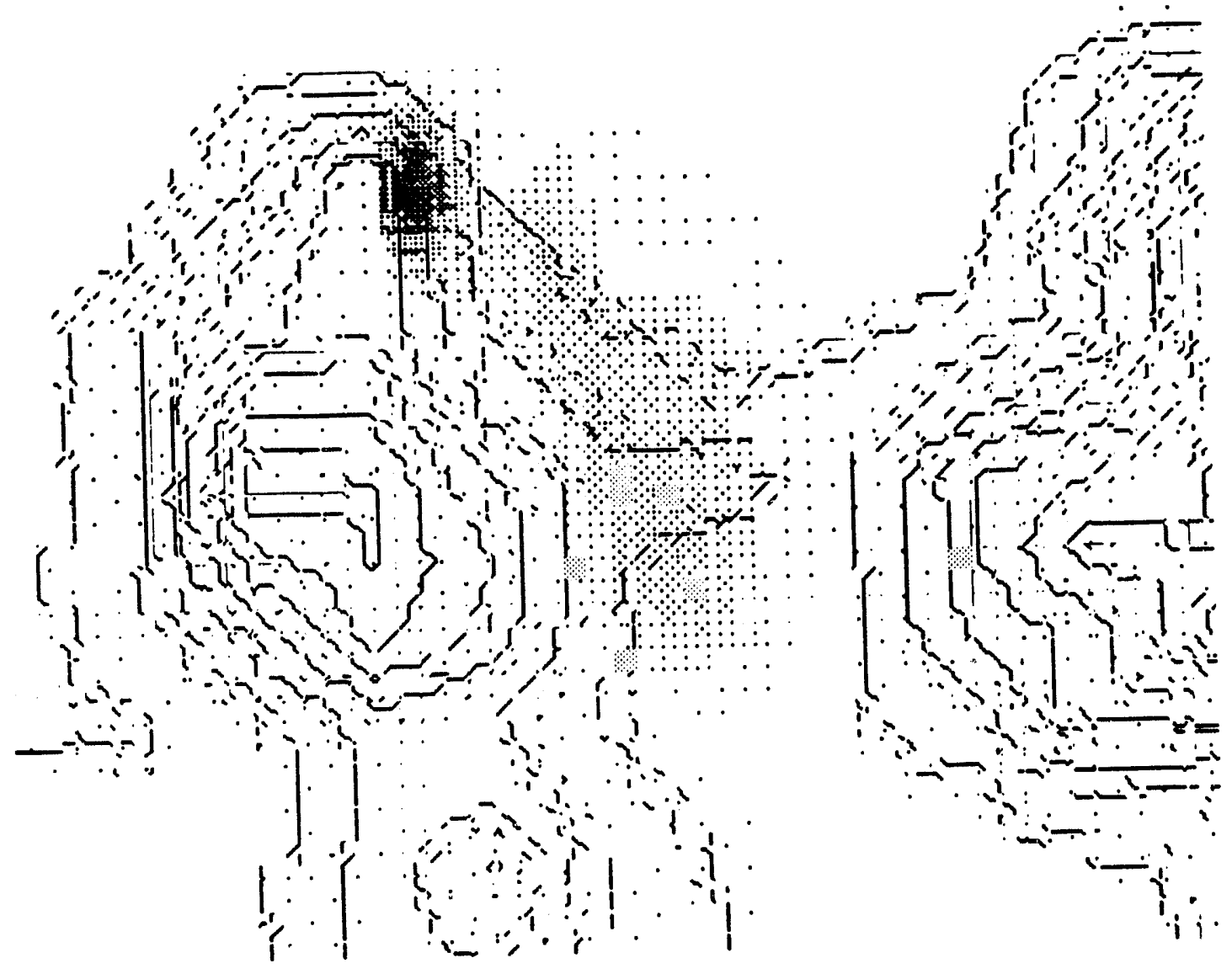

Figure 8. Sample Animation Frame 

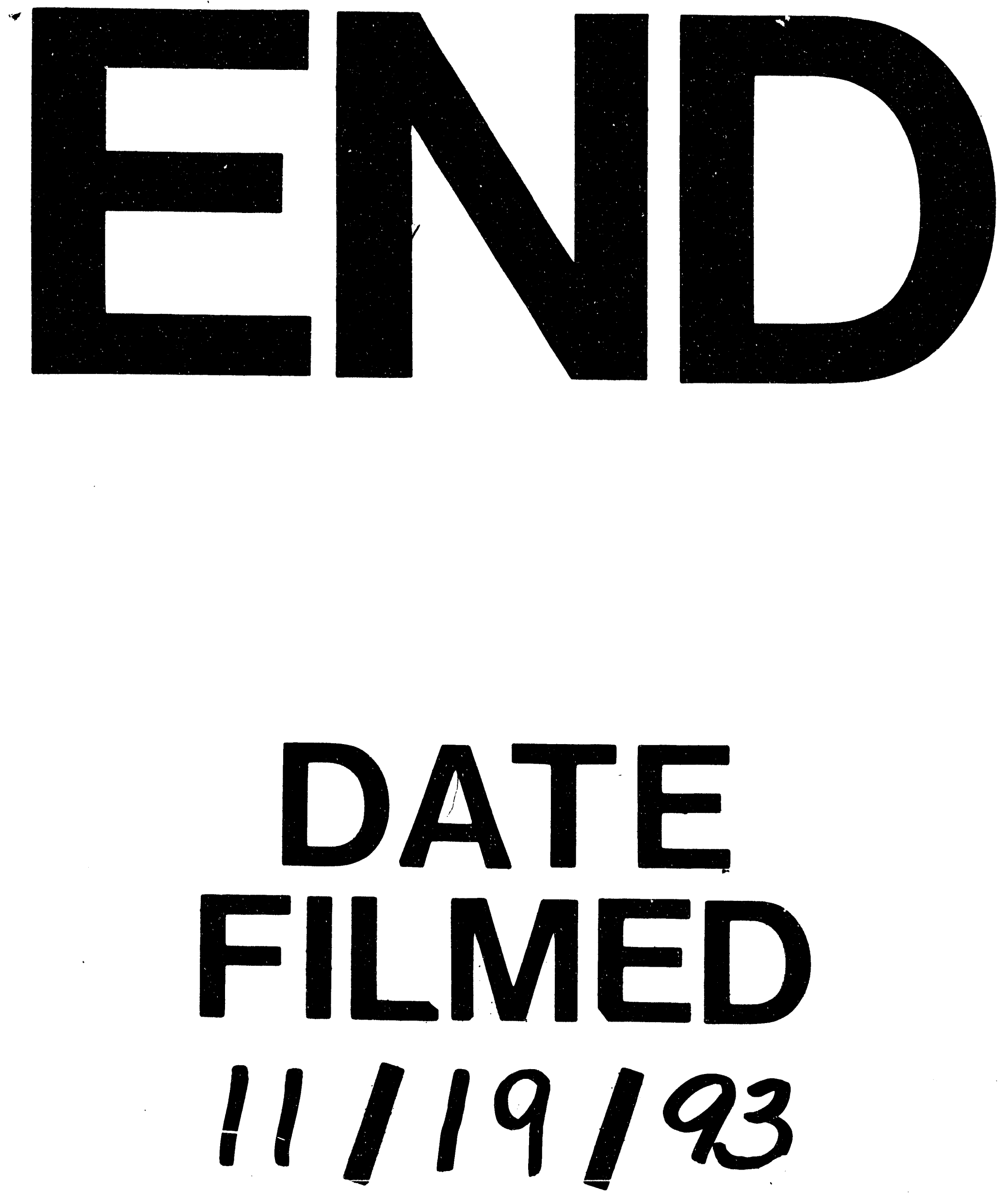

1 
